\title{
Sistematización de experiencias vividas en la I Feria de Simulación y Análisis de Sistemas de Costos*
}

\section{Systemization of experiences had been lived in the first fair systems simulation and analysis of cost experiences}

\section{Sistematização de experiências vividas na I Feria de simulação e análise de sistemas de custos}

\author{
Alicia Patricia Duque-Sánchez \\ Magíster en Contabilidad, Universidad de Medellín; Profesora tiempo completo de la Universidad del Atlántico, Barranquilla, Colombia. \\ aliciaduque@mail.uniatlantico.edu.co
}

F. Recepción: 1 julio 2016

F. Aceptación: 30 julio 2016

\begin{abstract}
Resumen
La Feria de Simulación y Análisis de Sistemas de Costos constituye una estrategia didáctica en la formación de los estudiantes de costos del programa de Contaduría Pública de la Universidad del Atlántico, enfocada al desarrollo de competencias comunicativas, profesionales y habilidades de orden superior. Corresponde a la socialización de los proyectos sobre la implementación de sistemas de costos, basados en la simulación de información de empresas reales. El artículo tiene el propósito de examinar con análisis crítico la primera versión de esa Feria, realizada en junio de 2016. El método utilizado fue el de sistematización de experiencias. Entre los resultados obtenidos se destaca la determinación de tres ejes centrales: Aporte de innovación, factores de éxito y aspectos a mejorar; con sus respectivas manifestaciones, causas y conclusiones. Una de las conclusiones derivadas de la sistematización de experiencias de la Feria, es que la simulación de los procesos productivos permite un acercamiento del estudiante con situaciones reales de las empresas industriales y el desarrollo de habilidades de orden superior, lo que a su vez genera la apropiación de conocimientos de los temas de contabilidad de costos.
\end{abstract}

\section{Palabras clave}

Sistematización de experiencias, simulación de sistemas de acumulación de costos, estrategia didáctica.

* Cómo citar: Duque-Sánchez, A. P. (2016). Sistematización de experiencias vividas en la I Feria de Simulación y Análisis de Sistemas de Costos. Revista Libre Empresa, 13(2), 85-101 http://dx.doi.org/10.18041/libemp.2016.v13n2.26206 


\section{Abstract}

Fair simulation and analysis of cost systems is a teaching strategy in the training of students cost Public Accounting program at the Universidad del Atlántico, focused on the development of communication skills, professional and higher order skills. It corresponds to the socialization of the projects on the implementation of cost systems based on simulation of real business information. This research aims to examine critically analyzes the first version of the Fair, on June, 2016. The method used was the systematization of experiences. Among the results determining highlighted three main components: Contribution of innovation, success factors and areas for improvement; with their respective manifestations, causes and conclusions. One of the conclusions derived from the systematization of the Fair, is that the simulation of production processes allows an approach student with real situations of industrial enterprises and the development of higher order skills which in turn generates the appropriation knowledge of cost accounting issues.

\section{Keywords}

Systemization of experiences, simulation cost accumulation systems, teaching strategy.

\section{Resumo}

Simulação e análise de sistemas de custos Fair é uma estratégia de ensino na formação de estudantes do custo do programa de Contabilidade Pública da Universidade do Atlântico, com foco no desenvolvimento de habilidades de comunicação, habilidades de ordem profissional e superior. Corresponde à socialização dos projetos sobre a implementação de sistemas de custos com base na simulação de informações de negócios real. O artigo tem por objetivo analisar criticamente a primeira versão da Feira, realizado em Junho de 2016. O método de análise utilizado foi a sistematização de experiências. Entre os resultados que determinam destacadas três componentes principais: Contribuição da inovação, fatores de sucesso e áreas de melhoria; com as respectivas manifestações, causas e conclusões. Uma das conclusões derivadas da sistematização da Feira, é que a simulação de processos de produção permite que um estudante de aproximação com situações reais de empresas industriais e o desenvolvimento de habilidades de ordem superior, que por sua vez gera o apropriação do conhecimento de questões de contabilidade de custos.

\section{Palavras chave}

Sistematizar experiências, sistemas de acumulação de custos de simulação, estratégia de ensino.

\section{Introducción}

La innovación en las estrategias didácticas es una tarea inacabada de los profesores que se esmeran por mejorar su práctica, para que el estudiante aprenda más y de mejor manera, con una formación integral; para lograr, además de ese aprendizaje significativo, que aprendan para la vida, provistos del arsenal de herramientas que constituyen las habilidades de orden superior, es por esto que el presente trabajo va en sintonía con esa dinámica de mejora continua. 
La Feria de Simulación y Análisis de Sistemas de Costos constituye una estrategia didáctica en la formación del contador público, enfocada al desarrollo de competencias comunicativas, profesionales (en el área de costos) y habilidades de orden superior, tales como: Pensamiento sistémico y creatividad. Corresponde a la socialización de los proyectos desarrollados por los estudiantes de Contaduría Pública de la Universidad del Atlántico, en los cursos de «Introducción a los Costos y Costos por Órdenes deProducción» y de «Costos por Procesos y Conjuntos» sobre la implementación de sistemas de costos, basados en la simulación de información de empresas reales.

Este trabajo presenta como objetivo general de investigación: Examinar la I Feria de Simulación y Análisis de Sistemas de Costos realizada el 10 de junio de 2016 en la Universidad del Atlántico; y aplica el método de sistematización de experiencias, el cual luego de desarrollarlo, el investigador resulta fortalecido al cumplir con los retos de análisis, síntesis, reflexión crítica, identificación de patrones, por lo que es altamente recomendable su utilización. El proceso de sistematización de experiencias "tiene el propósito final de que tanto quienes han estado involucrados en el proceso, como otras/ os puedan aprender de la experiencia” (Navarro \& Roche, 2013, p. 15).

Un aporte valioso para promover la práctica de la sistematización de experiencias en profesores y estudiantes en la educación superior, tomando lo que expresó Jara en (De La Cruz, 2010, p. 9), radica en que su realización contribuye a "fortalecer las capacidades transformadoras de los sujetos sociales", lo que contribuye a su vez, al desarrollo del pensamiento crítico y del pensamiento sistémico, habilidades de orden superior requeridas en la educación del siglo XXI (Hepp, 2009).

Esto se manifiesta, por un lado, con el pensamiento crítico que se ostenta en el énfasis de la interpretación crítica que hace parte del proceso de sistematización. Y por otro lado, el pensamiento sistémico que se pone a prueba en las condiciones personales que plantea Jara (1994b), que se requiere para hacer posible el ejercicio de sistematización de experiencias, en especial la concerniente con la "sensibilidad para dejar hablar la experiencia por sí misma”.

\section{Marco teórico}

La presente investigación se sustentó en posturas epistemológicas extraídas de la Teoría Crítica y la Teoría de Sistemas.

De la Teoría Crítica se toman argumentos de Jürgen Habermas sobre la acción comunicativa, que va acorde con la Pedagogía Crítica con respecto al empoderamiento impulsado por Paulo Freire; todo esto desemboca en el aspecto cualitativo de la investigación, caracterizado por la intervención del investigador y, por otro lado, en el desarrollo del pensamiento crítico y la interpretación crítica, importantes para la realización de la sistematización de experiencias, que es promovida por Óscar Jara Holliday.

En cuanto a la Teoría de Sistemas se toma el aspecto del pensamiento sistémico que se desprende de su rama denominada cibernética de segundo orden. 
Todo lo anterior, para destacar la importancia y el surgimiento de las habilidades de orden superior: pensamiento crítico y pensamiento sistémico, en estudiantes y profesores universitarios; que se ven fortalecidas a través del ejercicio de la estrategia didáctica de socialización de proyectos de implementación de sistemas de acumulación de costos, y también, en la respectiva sistematización de esa experiencia didáctica, expuesta en el presente trabajo académico.

A continuación un recorrido con los aspectos a destacar sobre sistematización de experiencias, pensamiento crítico, pensamiento sistémico, y su relación con la Teoría Crítica y la Teoría de Sistemas:

Jara (1994b, p. 131) define la sistematización de experiencias como

... aquella interpretación crítica de una o varias experiencias, que, a partir de su ordenamiento y reconstrucción, descubre o explicita la lógica del proceso vivido, los factores que han intervenido en dicho proceso, cómo se han relacionado entre sí, y por qué lo han hecho de ese modo.

Esta definición, vislumbra las características esenciales de la sistematización de experiencias: la interpretación crítica y la identificación de la lógica del proceso; que precisamente preocupan a Jara que no sean tenidas en cuenta por aquellos quienes, en la tendencia de instrumentalizar este método de investigación cualitativa, lo implementen simplemente con un enfoque de ordenamiento y reconstrucción de información (De la Cruz, 2010).

Agrega Jara (1994b) que es indispensable para sistematizar el pensar dinámico, riguroso, procesual, crítico y creativo. Esto implica fortalecer habilidades de orden superior, entre ellas el pensamiento crítico y el pensamiento sistémico.

El pensamiento crítico se concibe como aquel que desarrolla en los estudiantes una óptima capacidad de juicio (Sanz de Acedo, 2013) (González, 2006), y se evidencia su relación con la pedagogía crítica porque según Tamayo (2006), citado por Corredor \& López, (2013), ésta ayuda a enfatizar en una educación para el pensamiento crítico, en su formación en la reflexión argumentada, el análisis y la investigación de la realidad.

Otro aspecto a destacar es que la pedagogía crítica intenta, desde la enseñanza, empoderar a los estudiantes para que sean artífices de sus vidas y puedan desarrollar su autonomía (Gómez \& Gómez, 2011); asunto importante para el fortalecimiento de habilidades de orden superior.

Este empoderamiento va en sintonía con reflexiones de Paulo Freire que, en su obra “Pedagogía del Oprimido” publicada en los años 70’s, deja ver las relaciones que se tejen entre la teoría crítica y la pedagogía crítica latinoamericana (Gómez \& Gómez, 2011).

Con respecto a la Teoría Crítica se destacan los aportes de Habermas, sobre el papel de la acción comunicativa y la correspondiente racionalidad, utilizando la Teoría de Sistemas 
para explicar cómo la racionalización del mundo de la vida permite otro paso en la evolución social (Noguera, 1996).

Habermas distingue la acción comunicativa (la que pretende motivar racionalmente basado en la vinculación/compromiso) de la acción estratégica en la argumentación (la que busca influir en el otro a través de la manipulación) (González, 2006); siendo relevante la acción comunicativa en el óptimo desarrollo de la sistematización de experiencias.

Por último, el pensamiento sistémico tomado como aquel "que se basa en la percepción del mundo real en términos de totalidades para su análisis, comprensión y guía de la acción” (Sobremonte, 2012, p. 289) tiene un notable desarrollo en la década de los 60’s, al surgir la cibernética de segundo orden, que centra su estudio en la relación entre el observador y el sistema sometido a observación, que con el trabajo de Heinz von Foerster establece que "no es posible separar a los observadores de los sistemas que están describiendo" (O’Connor \& Mc Dermott, 1998, p.276), situación que se hace evidente en la sistematización de experiencias.

\section{Metodología}

El presente documento se deriva de una investigación con enfoque cualitativo, con perspectiva participativa, bajo la modalidad de sistematización de experiencias.

Se desarrollaron las pautas generales de la propuesta metodológica para sistematizar experiencias presentadas inicialmente por Jara (1994b) y actualizada por la Fundación SES (2002) (Jara, 2006); que ha sido acogida por estudiosos de la temática como método de investigación cualitativa, en donde el profesor no solo es objeto de conocimiento sino sujeto de conocimiento y transformación (De la Cruz, 2010).

Entonces, se tomaron los aspectos básicos expuestos por Jara; mientras que sus especificidades, por corresponder a experiencias resultado de una estrategia didáctica, se basaron en lo expuesto por autores tales como Pérez (2016) (Navarro \& Roche, 2013), que decantan la sistematización de experiencias educativas en el contexto de la educación superior.

La propuesta metodológica consta de los siguientes cinco tiempos:

1. El punto de partida

2. Las preguntas iniciales

3. Recuperación del proceso vivido

4. La reflexión de fondo

5. Los puntos de llegada

Cada uno de estos tiempos planteados se subdividen en momentos (o elementos) constitutivos, que se van a indicar a medida que se va exponiendo el proceso de sistematización de las experiencias objeto de estudio.

A continuación se muestra el desarrollo de la sistematización de experiencias. 


\section{El punto de partida}

1.1 Haber participado de la experiencia. Todo el equipo de sistematización de experiencias participó en la I Feria de simulación y análisis de sistemas de costos; de una manera heterogénea, lo que permitió una diversidad de puntos de vista al análisis crítico de las experiencias

1.2 Tener registros de las experiencias. Antes de iniciar el proceso de sistematización se tenían los registros escritos de las experiencias vividas de treinta (30) estudiantes que participaron en los stands de la Feria, 189 fotografías que registran desde la preparación hasta el desarrollo del evento, una presentación que se subió a internet en http://www. slideshare.net/profealiciaduque5/i-feria-de-simulaciony-analisis-de-sistema-de-costosuniatlantico-junio-10-de-2016 y un video de 18 minutos que abarca un recorrido por los stands y algunas participaciones de estudiantes en el desarrollo de la Feria.

\section{Las preguntas iniciales:}

2.1 Objetivo. Se estableció como objetivo de la sistematización:

Examinar la I Feria de Simulación y Análisis de Sistemas de Costos realizada el 10 de junio de 2016 en la Universidad del Atlántico; para compartir los resultados obtenidos con estudiantes y profesores de la Universidad del Atlántico, y de otras universidades; especialmente del área de costos.

2.2 Objeto. Las experiencias a sistematizar correspondieron a aquellas de quienes hicieron parte de la I Feria de Simulación y Análisis de Sistemas de Costos, realizada en la tarde noche del viernes 10 de junio de 2016 en el Lobby del Centro de convenciones de la Universidad del Atlántico.

2.3 Ejes de sistematización. Con base en las experiencias escritas analizadas, el criterio del equipo que realiza el proceso de sistematización y teniendo en cuenta el objetivo general del proyecto de investigación se determinaron los tres ejes centrales de las experiencias vividas en la Feria:

2.3.1 Aporte de innovación propio de la Feria. Por lo novedoso de la estrategia didáctica en el área de costos del programa de Contaduría Pública en la Universidad del Atlántico.

2.3.2 Factores que incidieron en el éxito de la Feria. Esto se manifiesta en la mayoría de las experiencias escritas, y es importante analizarlo.

2.3.3 Aspectos a mejorar en las siguientes versiones de la Feria. En algunas experiencias escritas se indican las fallas que se presentaron; esto es supremamente importante para determinar mejoras en las próximas versiones de la Feria.

\subsection{Fuentes de información}

Durante todo el proceso de sistematización de experiencias se contó con: dos tipos de fuente de información. Escritas y de participación directa.

Para mejorar la documentación sobre las experiencias de la Feria que ya se tenía disponible, durante el proceso de sistematización, se realizó lo siguiente: 
a) Envío de solicitud por correo electrónico de las experiencias vividas de los cinco (5) profesores invitados que asistieron. Se recibieron las experiencias escritas de dos (2) profesores que asistieron a la I Feria de Simulación y Análisis de Sistemas de Costos.

b) Envío de solicitud por correo electrónico, a los estudiantes que habían enviado sus experiencias vividas, de ampliar más en algunos apartes de lo que habían escrito, que no estaban claros o que no habían sido lo suficientemente específicos. Contestaron el 37\%, muy posiblemente porque se encontraban en período de vacaciones de mitad de año.

c) Se contó con la participación directa de dos equipos pequeños de trabajo de apoyo que laboraron con la Coordinadora de Sistematización en tiempos y lugares diferentes. El primer equipo conformado por las dos estudiantes universitarias que colaboraron en la logística del evento (entrega de botones, toma de fotos y demás), y el segundo, integrado por dos estudiantes de Contaduría Pública que hicieron parte de un stand de la Feria.

2.5 Procedimiento a seguir

Se elaboró un plan para la sistematización que fue organizado en tres etapas y acoge a los cinco tiempos planeados por Jara.

\section{Recuperación del proceso vivido}

3.1 Reconstrucción histórica de la experiencia

La I Feria de Simulación y Análisis de Sistemas de Costos, por constituir el primer evento académico de esta naturaleza a realizarse en el programa de Contaduría Pública de la Universidad del Atlántico causó en primera instancia temor por la realización del mismo, por parte de los estudiantes, pero, gracias al entusiasmo y persistencia de la profesora a cargo, y con el apoyo motivacional de la película recomendada: "Los inventores", ${ }^{1}$ los estudiantes aceptaron el reto, animándose los unos a los otros, con responsabilidad y dedicación (procurando dar lo mejor), con mucha creatividad.

Resultó exitosa la realización del evento, aunque con algunos inconvenientes que se espera mejorar en las próximas versiones.

3.2 Ordenación y clasificación de la experiencia con base en los ejes de sistematización Esta parte del proceso se da en la primera sesión del equipo encargado de la sistematización de las experiencias, radica en descomponer las experiencias escritas, en fragmentos; para ir identificándolos con alguno de los tres ejes de sistematización. Pueden resultar comentarios de experiencias que no se apliquen a ninguno de los tres ejes, eso se aparta de los pasos siguientes; pero, son retomados posteriormente por si se encuentra algún aspecto relacionado.

Con esto, el equipo encargado de la sistematización se familiariza con lo que se incluyen en las experiencias y se determina el número de las repeticiones (frecuencia) de las manifestaciones de los diferentes aspectos para cada uno de los ejes centrales de sistematización.

1 Spare Parts (La vida robot) conocida también como Los inventores, es una película estadounidense estrenada en 2015, dirigida por Sean McNamara y producida por David Alpert, Rick Jacobs, Leslie Kolins Small, George Lopez, y Ben Odell. Está basada en una historia real; trata de un grupo de estudiantes hispanos ilegales de una escuela de secundaria pública en los Estados Unidos, que sin tener ningún tipo de experiencia en la temática y con muy escasos recursos económicos, motivados por un sueño, aplicando mucha creatividad, los conocimientos y cualidades de cada miembro del equipo, participan y ganan en un concurso de robótica, superando a estudiantes de universidades de alto prestigio en Estados Unidos como el MIT. 
Luego, se toman los fragmentos más representativos de los aspectos manifestados que más se repiten, o que se consideren más valiosos para el equipo que sistematiza (aunque no sea frecuentemente repetida).

En otra sesión (reunión de otro día) se revisa lo realizado hasta el momento, y se ajusta donde corresponda, es por esto que el conocimiento es espiral y no lineal. Se evalúa varias veces, hasta definir plenamente los aspectos característicos con que se manifiestan las experiencias en los diferentes ejes, mejorando cada vez la redacción y la ubicación en cada eje. Porque en la sesión inicial algunos aspectos se consideraron en un eje, y en la siguiente sesión se ubicaron mejor en otro, según ciertos argumentos de criterios de análisis, más o menos elaborados.

\section{La reflexión de fondo}

\subsection{Analizar y sintetizar}

Se analiza con una reflexión más profunda el listado de la clasificación de las diferentes manifestaciones para cada uno de los tres ejes y, luego se redacta, un resumen de cada uno de los ejes centrales.

La clasificación inicial con las manifestaciones, y el resumen de cada eje permite una interpretación crítica de lo vivido y, superar la descripción de los hechos sucedidos. Esto se somete a consideración de un nuevo equipo de trabajo, en otro día, otro lugar, de manera que se profundice más la reflexión.

Como resultado de las dos reuniones con el primer equipo de sistematización y luego validada y ajustada por el segundo equipo de trabajo se determinaron los siguientes aspectos de manifestaciones, que se consolida en la Tabla 1, en la que se indica las manifestaciones de:

- Los aportes de innovación propios de la Feria

- Los factores de éxito de la Feria

- Los aspectos a mejorar en la nuevas versiones de la Feria

4.2 Hacer una interpretación crítica del proceso

Tomando como base lo incluido en la Tabla 1, y las discusiones de los equipos de sistematización, como resultado de la interpretación crítica de la inter-relación de los aspectos clasificados, se identificaron las causas de cada manifestación, lo cual generó la sintetización de la información en un cuadro por cada eje central.

\section{Los puntos de llegada}

\subsection{Formulación de conclusiones}

Con base en los tres cuadros obtenidos (manifestaciones con sus causas, para cada eje central) se colocaron flechas que relacionaban las causas de cada una de las manifestaciones indicadas. Con esto se logró identificar los nudos críticos, es decir, las manifestaciones o causas que más relaciones tienen (desde donde salen o llegan las flechas), y luego, con una reflexión crítica, se redactaron conclusiones. Esto aparece sintetizado en las Tablas 2, 3 y 4. 


\section{Tabla 1.}

Manifestaciones ( $M$ ) identificadas en las experiencias vividas y ubicadas en cada eje central

\begin{tabular}{|c|c|}
\hline $\begin{array}{l}\text { Eje 1: Aportes de innovación } \\
\text { propios de la Feria }\end{array}$ & $\begin{array}{l}\text { Eje 2: Factores de éxito de } \\
\text { la Feria }\end{array}$ \\
\hline \multirow{2}{*}{$\begin{array}{l}\text { M1. Proyecta la visualización } \\
\text { del estudiante como asesor de } \\
\text { costos (en su futuro profesional) }\end{array}$} & $\begin{array}{l}\text { M1. Cumplimiento de los objetivos } \\
\text { planteados en la Feria }\end{array}$ \\
\hline & $\begin{array}{l}\text { M2. Enseñanzas y sugerencias de } \\
\text { los profesores invitados }\end{array}$ \\
\hline \multirow{3}{*}{$\begin{array}{l}\text { M2. Simulación de procesos } \\
\text { productivos }\end{array}$} & $\begin{array}{l}\text { M3. Se logró aprendizaje } \\
\text { significativo en las temáticas del } \\
\text { área de costos }\end{array}$ \\
\hline & $\begin{array}{l}\text { M4. Alcanza un acercamiento a } \\
\text { situaciones reales de las empresas } \\
\text { industriales }\end{array}$ \\
\hline & $\begin{array}{l}\text { M5. Evidencia el desarrollo de las } \\
\text { habilidades de orden superior }\end{array}$ \\
\hline \multirow{2}{*}{$\begin{array}{l}\text { M3. Intercambio de roles } \\
\text { (expositor y visitante) }\end{array}$} & $\begin{array}{l}\text { M6. Permite demostrar } \\
\text { competencias comunicativas }\end{array}$ \\
\hline & M7. Trabajo en equipo \\
\hline \multirow{4}{*}{$\begin{array}{l}\text { M4. La socialización de proyectos } \\
\text { de diferentes grupos y cursos de } \\
\text { costos (horario diurno y nocturno) } \\
\text { del Programa de Contaduría } \\
\text { Pública }\end{array}$} & M8. Motivación de estudiantes \\
\hline & M9. Organización del evento \\
\hline & M10. Dedicación \\
\hline & M11. Espacio amplio de Integración \\
\hline \multirow{2}{*}{$\begin{array}{l}\text { M5. Varios profesores del área de } \\
\text { costo visitaron la Feria }\end{array}$} & $\begin{array}{l}\text { M12. Estudiantes también se } \\
\text { ponen en contacto con la temática } \\
\text { que verán el siguiente semestre }\end{array}$ \\
\hline & $\begin{array}{l}\text { M13. La realización de esta } \\
\text { Feria deja un referente para los } \\
\text { estudiantes en lo que será la } \\
\text { próxima feria }\end{array}$ \\
\hline
\end{tabular}

Eje 3: Aspectos a mejorar para próximas versiones de la Feria

M1. Insuficiente ventilación del área de exposición

M2. Sistema eléctrico deficiente

M3. Faltó iluminación de ciertas áreas

M4. Faltó retroalimentación en clase

M5. No se evidenció la presentación de los proyectos con la nueva normativa contable

M6. Resultó insuficiente los profesores invitados

M7. Faltó más tiempo de anticipación para preparar la Feria

M8. Algunos estudiantes no estaban suficientemente preparados

M9. Se hizo poco control de la asistencia de expositores (incluye llegada a la Feria) y rotación de roles

M10. Faltó retroalimentación de la profesora, mientras recorría los stand de la Feria

M11. Faltó publicidad del evento

M12. No estaban todas las mesas en el lugar del evento

Fuente: Elaboración propia como producto de la sistematización de experiencias

Las conclusiones incluyeron las lecciones aprendidas. ${ }^{2}$ Estas, posteriormente fueron revisadas por los integrantes del segundo pequeño equipo de sistematización, realizando algunos ajustes producto de la revisión de cada uno de las Tablas obtenidas y las inter-

2 Lecciones aprendidas son los aprendizajes que es posible extraer de la reflexión y el análisis realizados. 
relaciones que dan cuenta de las causas de cada una de las manifestaciones que habían sido expresadas directa o indirectamente en las experiencias escritas.

Las manifestaciones, causas y conclusiones de los aportes de innovación propios de la Feria (eje central 1) se encuentran sintetizadas en la Tabla 2, en donde se relaciona a través de unas flechas partiendo de la causa hasta llegar a la manifestación.

\section{Tabla 2.}

Manifestaciones, causas y tendencias del eje central 1. Aportes de innovación propios de la Feria

\begin{tabular}{|c|c|c|}
\hline Manifestaciones ( M ) & Causas ( C ) & Conclusiones \\
\hline $\begin{array}{l}\text { M1. Proyecta la visualización } \\
\text { del estudiante como asesor } \\
\text { de costos (en su futuro } \\
\text { profesional) }\end{array}$ & $\begin{array}{l}\text { C1. Botón como "asesores de } \\
\text { costos" se les colocó a medida } \\
\text { que llegaban los grupos de } \\
\text { expositores }\end{array}$ & $\begin{array}{l}\text { El desempeño de roles de los diferentes } \\
\text { perfiles del Contador Público, como } \\
\text { estrategia didáctica, permite al } \\
\text { estudiante ir familiarizándose más }\end{array}$ \\
\hline $\begin{array}{l}\text { M2. La simulación de procesos } \\
\text { productivos }\end{array}$ & $\begin{array}{l}\text { C2. Código de vestimenta } \\
\text { elegante propio de los "asesores } \\
\text { de costos" }\end{array}$ & $\begin{array}{l}\text { y mejor con estos, a lo largo de su } \\
\text { formación profesional; lo que contribuye } \\
\text { a que vaya poco a poco considerando }\end{array}$ \\
\hline $\begin{array}{l}\text { M3. Intercambio de roles } \\
\text { (expositor y visitante) }\end{array}$ & $\begin{array}{l}\text { C3. Estrategias ideadas por la } \\
\text { profesora organizadora de la Feria }\end{array}$ & $\begin{array}{l}\text { uno u otro perfil, en su proyecto de vida } \\
\text { profesional. }\end{array}$ \\
\hline $\begin{array}{l}\text { M4. La socialización de } \\
\text { proyectos de diferentes } \\
\text { grupos y cursos de costos } \\
\text { (horario diurno y nocturno) } \\
\text { del Programa de Contaduría } \\
\text { Pública }\end{array}$ & & $\begin{array}{l}\text { Las nuevas estrategias docentes } \\
\text { puestas en práctica en los diferentes } \\
\text { eventos, deben ser compartidas a la } \\
\text { comunidad académica, para motivar e } \\
\text { inspirar a que otros profesores aporten } \\
\text { con nuevas propuestas que fortalezcan }\end{array}$ \\
\hline $\begin{array}{l}\text { M5. Varios profesores del área } \\
\text { de costo visitaron la Feria }\end{array}$ & & $\begin{array}{l}\text { la buena imagen del programa de } \\
\text { Contaduría Pública, y en general de la } \\
\text { Universidad del Atlántico. }\end{array}$ \\
\hline
\end{tabular}

Fuente: Elaboración propia como producto del proceso de sistematización de experiencias

Todas las manifestaciones del eje central 1 fueron obtenidas de las interpretaciones realizadas por el primer equipo de trabajo, a partir de las experiencias escritas por los estudiantes. Estas interpretaciones dan respuesta a qué es lo que el estudiante pretendía decir, porque no aparecían muy claras, y fueron objeto de varias rondas de revisión y ajuste por parte del primer pequeño equipo, con una participación activa de la Coordinadora de sistematización; hasta quedar todas de acuerdo con lo obtenido. Lo que permitió un interesante ejercicio de análisis, reflexión y argumentación.

Las manifestaciones, causas y conclusiones de los factores de éxito de la Feria (eje central 2) se hallan sintetizadas en la Tabla 3. 
Tabla 3.

Manifestaciones (M), Causas (c) y Conclusiones del eje central 2. Factores de éxito de la Feria

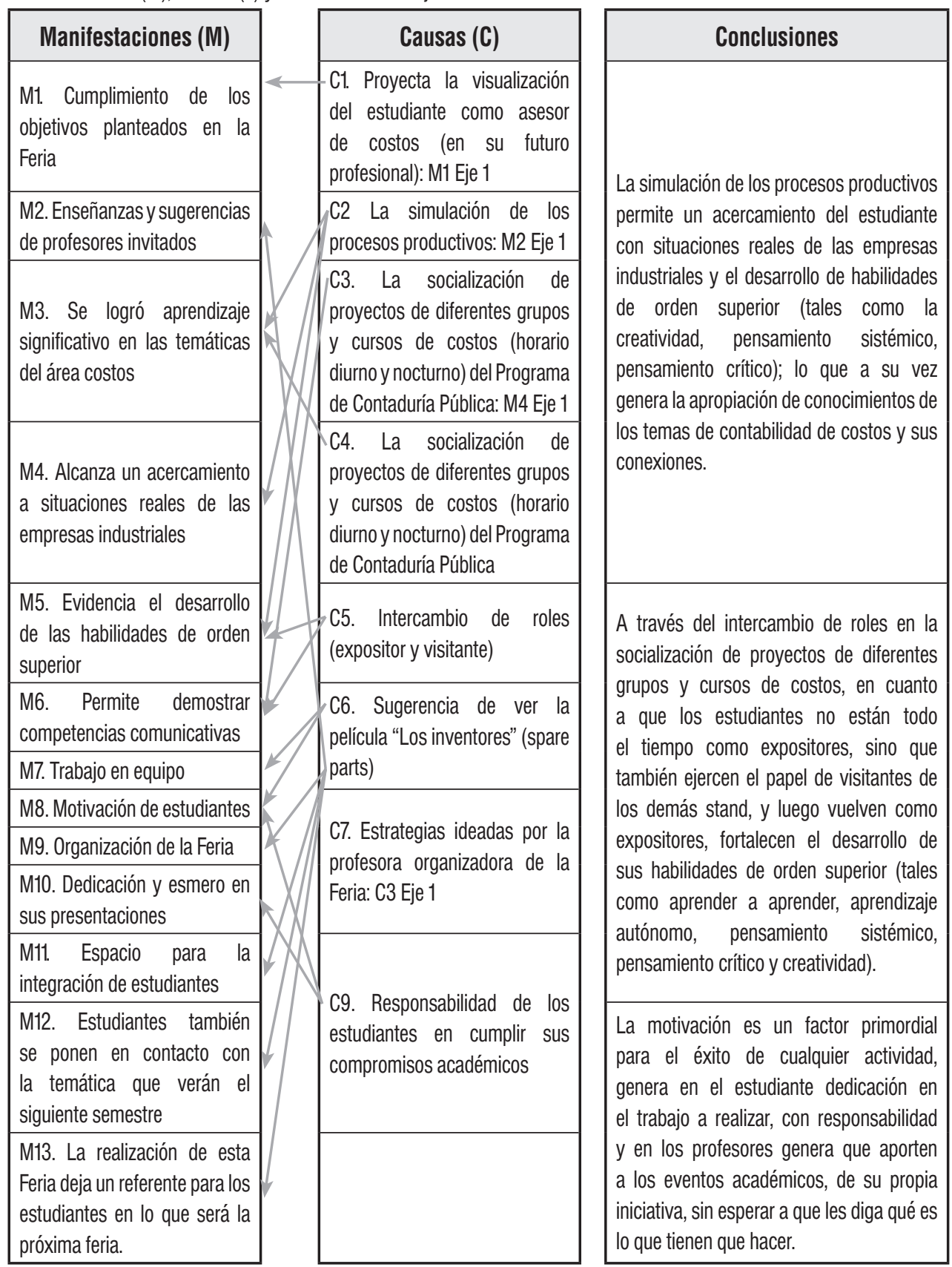

Fuente: Elaboración propia como producto del proceso de sistematización de experiencias 
Algunas de las manifestaciones incluidas en el eje central 2 fueron sugeridas por la profesora Alicia Duque, en su calidad de Coordinadora del evento. La mayoría, fueron indicadas en las experiencias escritas por los estudiantes, entonces, el primer equipo de trabajo se encargó de re-expresarlas a manera de síntesis, resultando menos complejo el proceso de identificación de manifestaciones y sus causas en este eje enfocado a los factores de éxito de la Feria.

Tres de las manifestaciones incluidas en la Tabla 2 (como parte del eje de aportes de innovación propios de la Feria) aparecen como causas en la Tabla 3 (del eje de factores de éxito de la Feria), y una de las causas incluida en la Tabla 2 aparece también como causa para factores de éxito de la Feria. Estas interrelaciones fueron bien estudiadas y hubo una pequeña dificultad en ubicar algunas flechas, y por eso fue muy importante la participación del segundo pequeño grupo, quien colaboró en organizarlas de mejor manera. Las manifestaciones, causas y conclusiones de los aspectos a mejorar para las próximas versiones de la Feria (eje central 3) se hallan sintetizadas en la Tabla 4.

Las manifestaciones incluidas en el eje central 3 excepto la identificada como número 9, fueron expresadas en las experiencias escritas por pocos estudiantes; porque en su mayoría, se dedicaron a resaltar los aspectos positivos de la Feria. Entonces, el primer equipo las identificó y las organizó adecuadamente.

Por último, retomando algunas ideas discutidas al reconstruir las experiencias de la Feria, durante su proceso de interpretación y con base en un análisis reflexivo, crítico y riguroso, se establecieron algunas recomendaciones, para futuras versiones de la Feria de Simulación y Análisis de Sistemas de Costos.

\subsection{Comunicar los aprendizajes}

Como resultado del proceso de sistematización, el primer equipo de sistematización escribió sobre los aprendizajes y experiencias que tuvieron, no de la Feria, sino del proceso mismo de sistematización; en el que se destaca que desarrollaron su pensamiento crítico. He aquí algunos fragmentos de lo escrito:

“... el proceso de sistematización de experiencias me dio la oportunidad de entender qué se hace y para qué se hace, lo que le da un sentido crítico a los diferentes análisis realizados, y aunque hubo momentos que me sentía perdida, con la ayuda de Alicia y de mi compañera de equipo me ayudaron a encontrar nuevamente el cauce y el sentido para continuar los análisis"... : (Colaboradora 1, del primer pequeño equipo).

... "fue interesante darse cuenta de las múltiples interpretaciones a partir de un mismo escrito de experiencia y cómo lograr definir(escoger) una, con la ayuda de varios elementos de juicios y teniendo claro a dónde queríamos llegar con los ejes". (Colaboradora 1, del primer pequeño equipo).

... "desarrollamos nuestro proceso de relacionar varias partes de experiencias que habíamos leído que nos habían llamado la atención, por la forma en que estaban escritas o lo que decían". (Colaboradora 2, del primer pequeño equipo). 
Tabla 4.

Manifestaciones, causas y tendencias del eje central 3. Aspectos a mejorar para próximas versiones de la Feria

\begin{tabular}{|c|c|c|}
\hline Manifestaciones & Causas & Conclusiones \\
\hline $\begin{array}{l}\text { M1. Insuficiente ventilación del } \\
\text { área de exposición }\end{array}$ & C1. Factores Institucionales que están & La Universidad del Atlántico \\
\hline M2. Sistema eléctrico deficiente & & acreditación institucional, que \\
\hline $\begin{array}{l}\text { M3. Faltó iluminación de ciertas } \\
\text { áreas }\end{array}$ & C2. No se previó una retroalimentación & $\begin{array}{l}\text { incluye mejoramiento de toda su } \\
\text { infraestructura física y el apoyo }\end{array}$ \\
\hline $\begin{array}{l}\text { M4. Faltó retroalimentación en } \\
\text { clase }\end{array}$ & $\begin{array}{l}\text { en clase al momento de programar la } \\
\text { fecha de la Feria. }\end{array}$ & $\begin{array}{l}\text { a la reallzacion de eventos con } \\
\text { iniciativa de los profesores, en } \\
\text { desarrollo de sus estrategias }\end{array}$ \\
\hline $\begin{array}{l}\text { M5. No se evidenció la } \\
\text { presentación de los proyectos } \\
\text { con la nueva normativa contable }\end{array}$ & $\begin{array}{l}\text { C3. Durante clases se mencionó } \\
\text { aspectos de la nueva normativa } \\
\text { contable en la temática de contabilidad }\end{array}$ & $\begin{array}{l}\text { pedagógicas. También abarca la } \\
\text { formalización de estos eventos, } \\
\text { para que sean abiertos a toda la }\end{array}$ \\
\hline $\begin{array}{l}\text { M6. Resultó insuficiente los } \\
\text { profesores invitados }\end{array}$ & $\begin{array}{l}\text { de costos, pero no se profundizó } \\
\text { mucho en esto. }\end{array}$ & $\begin{array}{l}\text { comunidad academica. Poco a } \\
\text { poco se ha venido preparando } \\
\text { para la realización de ferias }\end{array}$ \\
\hline $\begin{array}{l}\text { M7. Faltó más tiempo de } \\
\text { anticipación para preparar la } \\
\text { Feria }\end{array}$ & $\begin{array}{l}\text { C4. Se realizó la invitación a más } \\
\text { profesores, pero desafortunadamente } \\
\text { no todos pudieron asistir a la Feria. }\end{array}$ & $\begin{array}{l}\text { estudiantiles, como las del } \\
\text { Programa de Química y la de la } \\
\text { Facultad de ingenierías. Se están }\end{array}$ \\
\hline $\begin{array}{l}\text { M8. Algunos estudiantes } \\
\text { no estaban suficientemente } \\
\text { preparados }\end{array}$ & $\begin{array}{l}\text { C5. La planificación de clases se } \\
\text { enfocó en dar primero los temas de los } \\
\text { cursos, luego la implementación del }\end{array}$ & $\begin{array}{l}\text { estableciendo procealmientos } \\
\text { para separar los salones y el } \\
\text { préstamo de sillas y mesas. }\end{array}$ \\
\hline $\begin{array}{l}\text { M9. Se hizo poco control de } \\
\text { la asistencia de expositores }\end{array}$ & $\begin{array}{l}\text { sistema de costos, y finalmente la Feria } \\
\text { como socialización de los mismos }\end{array}$ & \\
\hline $\begin{array}{l}\text { (incluye llegada a la Feria) y } \\
\text { rotación de roles }\end{array}$ & C6. No se hizo una selección previa a & $\begin{array}{l}\text { Para mejorar la organización en } \\
\text { otras versiones de la Feria es }\end{array}$ \\
\hline $\begin{array}{l}\text { M10. Faltó retroalimentación de } \\
\text { la profesora mientras recorría los } \\
\text { stand de la Feria }\end{array}$ & $\begin{array}{l}\text { no hubieran terminado su proyecto de } \\
\text { implementación de sistemas de costos }\end{array}$ & $\begin{array}{l}\text { pertinente constitulr varıs comite, } \\
\text { dedicados a: Logística, publicidad, } \\
\text { control de asistencia, toma de }\end{array}$ \\
\hline M11. Faltó publicidad del evento & $\begin{array}{l}\text { C7. Por ser un evento sin antecedentes } \\
\text { en el Programa de Contaduría Pública, } \\
\text { la Coordinadora se hizo cargo de } \\
\text { gestionar y hacer seguimiento } \\
\text { de la logística del mismo, con la } \\
\text { colaboración de dos estudiantes } \\
\text { externas al Programa y de algunos } \\
\text { estudiantes participantes de la Feria. } \\
\text { Hizo falta más personal de apoyo. }\end{array}$ & $\begin{array}{l}\text { de apoyo. Estos comités estarán } \\
\text { integrados por estudiantes que } \\
\text { acepten de forma voluntaria hacer } \\
\text { parte de estos y adicionalmente } \\
\text { por los estudiantes, que no queden } \\
\text { seleccionados para exponer sus } \\
\text { proyectos en la Feria. }\end{array}$ \\
\hline $\begin{array}{l}\text { M12. No estaban todas las } \\
\text { mesas en el lugar del evento }\end{array}$ & $\begin{array}{l}\text { C8. El tiempo de realización de la Feria } \\
\text { de dos horas (5:30 pm a 7:30 pm) } \\
\text { no fue suficiente, y además empezó } \\
\text { media hora tarde. }\end{array}$ & $\begin{array}{l}\text { Es importante una selección previa } \\
\text { para determinar los grupos de } \\
\text { estudiantes que van a exponer en la } \\
\text { feria, con base a la presentación y } \\
\text { sustentaciones de sus proyectos en } \\
\text { el aula, de lo que tienen preparados } \\
\text { para presentar en el Feria. }\end{array}$ \\
\hline
\end{tabular}

Fuente: Elaboración propia como producto de la sistematización de experiencias 
Se destaca el avance significativo por parte del primer pequeño equipo de trabajo, que partió de las propias vivencias de la Feria, y del conjunto de escritos que expresan las experiencias vividas por parte de 30 participantes al evento; hasta lograr un primer documento sobre la respectiva sistematización de experiencias, que fue mejorado por el segundo pequeño equipo de trabajo.

\section{Resultado}

El resultado de la sistematización de experiencias lo constituye el informe del proceso de sistematización, que fue estructurado según lo indicado por Van de Velde (2008); conformado por:

1. Introducción

2. Descripción del proceso de sistematización de experiencias

3. Ilustración del papel personal de la Coordinadora de Sistematización, durante todo el proceso

4. Factores que incidieron positivamente en el proceso

5. Factores que incidieron negativamente en el proceso

6. Sugerencias de mejoras para nuevos procesos

7. Conclusiones

Los dos primeros puntos (introducción y descripción) ya se han mencionados en algunos apartes del presente documento, y los dos últimos (sugerencias de mejoras y conclusiones) serán expuestos en el punto de conclusiones del presente escrito, por lo tanto a continuación se destacan los principales aspectos del desarrollo de los puntos 3, 4 y 5 del informe del proceso de sistematización.

\section{Papel personal de la Coordinadora de Sistematización:}

El papel de la coordinadora del proceso de sistematización fue constituir los equipos de sistematización teniendo en cuenta las limitaciones de encontrarse en período de vacaciones de mitad de año, de guiarlos por las actividades a desarrollar e involucrarse en la realización de las mismas.

\section{Factores que incidieron positivamente en el proceso}

Los factores que incidieron positivamente en el proceso de sistematización fueron:

- De manera anticipada a la realización de la Feria, ya la profesora a cargo había solicitado a sus estudiantes que escribieran sobre las experiencias vividas en el evento, y la enviaran al correo electrónico de la Coordinadora de la Feria.

- Por ser de tipo voluntario, no todos respondieron a la solicitud. Sin embargo, se obtuvo una buena respuesta con treinta experiencias vividas enviadas al correo electrónico de la profesora, coordinadora de la Feria.

- Abundante literatura sobre el proceso de sistematización de experiencias.

- La gran disposición de ayuda de las integrantes de los equipos de sistematización, al aceptar el reto de involucrarse en un proceso desconocido para ellas, y de aprender a aprender. 
- Que la Coordinadora de Sistematización fuera la creadora y realizadora de la I Feria de Simulación y Análisis de Sistemas de Costos, permitió profundizar más en la identificación de las causas de las diferentes manifestaciones, para cada eje central.

\section{Factores que incidieron negativamente en el proceso}

Los factores que incidieron negativamente en el proceso de sistematización fueron:

- Falta de experiencia por parte de la Coordinadora, en la realización del proceso de sistematización.

- El período en que se realizó el proceso de sistematización de experiencias fue en las vacaciones de estudiantes de mitad de año.

\section{Conclusiones}

Las siguientes fueron las conclusiones obtenidas del proceso de sistematización de experiencias de la Feria:

- El desempeño de roles de los diferentes perfiles del Contador Público, como estrategia didáctica, permite al estudiante ir familiarizándose más y mejor con estos, a lo largo de su formación profesional; lo que contribuye a que vaya poco a poco considerando uno u otro perfil, como opción en su proyecto de vida profesional (Tabla 2).

- Las nuevas estrategias docentes puestas en práctica en los diferentes eventos, deben ser compartidas a la comunidad académica, para motivar e inspirar a que otros profesores aporten con nuevas propuestas, que propician una buena imagen del programa de Contaduría Pública, y en general de la Universidad del Atlántico (Tabla 2).

- La simulación de los procesos productivos permite un acercamiento del estudiante con situaciones reales de las empresas industriales y el desarrollo de habilidades de orden superior (tales como creatividad, pensamiento sistémico, pensamiento crítico); lo que a su vez genera la apropiación de conocimientos de los temas de contabilidad de costos y sus conexiones (Tabla 3 ).

- A través del intercambio de roles en la socialización de proyectos de diferentes grupos y cursos de costos, en cuanto a que los estudiantes no están todo el tiempo como expositores, sino que también ejercen el papel de visitantes de los demás stand, y luego vuelven como expositores, fortalecen el desarrollo de sus habilidades de orden superior (tales como aprender a aprender, aprendizaje autónomo, pensamiento sistémico, pensamiento crítico y creatividad), (Tabla 3).

- La motivación es un factor primordial para el éxito de cualquier actividad, genera en el estudiante dedicación en el trabajo a realizar, con responsabilidad y en los profesores que aporten a los eventos académicos, de su propia iniciativa, sin esperar a que se les diga qué es lo que tienen que hacer (Tabla 3).

- La Universidad del Atlántico se encuentra en un proceso de acreditación institucional, que incluye mejoramiento de toda su infraestructura física y el apoyo a la realización de eventos con iniciativa de los profesores, en desarrollo de sus estrategias didácticas.

También abarca la formalización de estos eventos, para que sean abiertos a toda la comunidad académica. Poco a poco se ha venido preparando para la realización de ferias 
estudiantiles, como las del Programa de Química y la de la Facultad de Ingenierías. Se están estableciendo procedimientos para separar los salones y el préstamo de sillas y mesas (Tabla 4).

- Es importante una selección previa para determinar los grupos de estudiantes que van a exponer en la Feria, con base a la presentación y sustentaciones de sus proyectos en el aula, de lo que tienen preparados para presentar en la Feria (Tabla 4).

- Para mejorar la organización en otras versiones de la Feria, es pertinente constituir varios comité, dedicados a: logística, publicidad, control de asistencia, toma de evidencias, entre otras actividades de apoyo. Estos comités estarán integrados por estudiantes que acepten de forma voluntaria hacer parte de estos y que no queden seleccionados para exponer sus proyectos en la Feria (Tabla 4).

Por último, una de las lecciones aprendidas producto del proceso de sistematización es que se aprende no solo de las experiencias objeto de sistematización, en las que los que participan la han vivido, sino también de lo experimentado en la realización propia del ejercicio mismo sistematización; lo que constituye una nueva experiencia.

\section{Conflicto de intereses}

La autora declara no tener ningún conflicto de intereses.

\section{Referencias bibliográficas}

1. Corredor, A., \& López, G. (2013). Pedagogía crítica en la educación superior: Una aproximación desde la didáctica. Revista Colombiana de Educación, 1(12), 166-194.

2. De la Cruz, A. (Julio de 2010). La sistematización de experiencias: aspectos teóricos y metodológicos. Entrevista a Óscar Jara. (I. d. Perú, Ed.) Revista de Investigación y Pedagogía Matinal, 4, 8-10.

3. Gómez, J., \& Gómez, L. (Enero-Julio de 2011). Elementos teóricos y prácticos de la pedagogía crítica: más allá de la educación, metáfora, escena y experiencia. (U. N. Rica, Ed.) Praxis, 181-190.

4. González, J. H. (2006). Discernimiento. Evolución del pensamiento crítico en la educación superior. Cali: Universidad ICESI.

5. Hepp, P. (Septiembre-Octubre de 2009). Transformar en compañía de los "nativos” del mundo digital. (M. M. Nacional, Ed.) Altablero, 52.

6. Jara, O. (1994a). Para sistematizar experiencias: una propuesta teórica y práctica. San José, Costa Rica: Alforja.

7. Jara, O. (1994b). Tres posibilidades de la sistematización. La Piragua, 129-135.

8. Jara, O. (2006). Guía para sistematizar experiencias. Mesoamérica: UICN- Programa Alianzas.

9. Navarro, A., \& Roche, A. M. (2013). Investigación en educación: Los procesos de sistematización de experiencias educativas. (U. N. Plata, Ed.) Oficios terrestres, 1(29).

10. Noguera, J. (1996). La teoría crítica: de Frankfurt a Habermas. (U. A. Barcelona, Ed.) Pap(Papers 50), 133-153. 
11. O’Connor, J., \& Mc Dermott, I. (1998). Introducción al pensamiento sistémico. Barcelona: Urano.

12. Pérez, T. (2016). Guía didáctica de Sistematización de Experiencias en contextos universitarios. Caracas, Venezuela: Universidad Nacional Abierta.

13. Sanz de Acedo, M. L. (2013). Competencias cognitivas en educación superior. Bogotá: Ediciones de la U.

14. SES, F. (2002). www.fundses.org.ar. Recuperado el 20 de 07 de 2016, de http://www. fundses.org.ar/archi/tematicas/sistematizacion/revista $\% 20$ sistematizacion $\% 20$ SES\% 202002.pdf

15. Sobremonte, E. (2012). Epistemología, teoría y modelos de intervención en Trabajo Social (Vol. 13). Bilbao, España: Universidad de Deusto.

16. Van de Velde, H. (2008). Sistematización. Estelí, Nicaragua: CICAP Centro de Investigación, Capacitación y Acción Pedagógica. 